\title{
Magnetic Resonance Imaging of Temperature Changes During Interstitial Microwave Heating: A Phantom Study
}

\section{A. Vitkin}

University of Toronto

\section{J. A. Moriarty}

University of Toronto

\section{R. D. Peters}

University of Toronto

\section{Michael C. Kolios}

University of Toronto

\section{A. S. Gladman}

University of Toronto

\section{digital.library.ryerson.ca/object/89}

\section{Please Cite:}

Vitkin, I. A., Moriarty, J. A., Peters, R. D., Kolios, M. C., Gladman, A. S., Chen, J. C., Hinks, R. S., Hunt, J. W., Wilson, B. C., Easty, A. C., Bronskill, M. J., Kucharczyk, W., Sherar, M. D., \& Henkelman, R. M. (1997). Magnetic resonance imaging of temperature changes during interstitial microwave heating: $A$ phantom study. Medical Physics, 24(2), 269-277.

doi:10.1118/1.598096

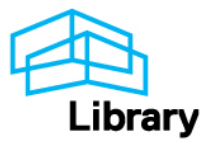




\section{Magnetic resonance imaging of temperature changes during interstitial microwave heating: A phantom study}

I. A. Vitkin ${ }^{\text {a) }}$

Ontario Cancer Institute/Princess Margaret Hospital, and Department of Medical Biophysics, University of Toronto, Toronto, Ontario M5G 2M9, Canada

J. A. Moriarty

Sunnybrook Health Science Centre, and Department of Medical Imaging, University of Toronto, Toronto, Ontario M4N 3M5, Canada

R. D. Peters

Sunnybrook Health Science Centre, and Department of Medical Biophysics, University of Toronto, Toronto, Ontario M4N 3M5, Canada

M. C. Kolios

Ontario Cancer Institute/Princess Margaret Hospital, and Department of Medical Biophysics, University of Toronto, Toronto, Ontario M5G 2M9, Canada

A. S. Gladman

Ontario Cancer Institute/Princess Margaret Hospital, and Institute of Biomedical Engineering, University of Toronto, Toronto, Ontario M5G 2M9, Canada

J. C. Chen

Sunnybrook Health Science Centre, and Department of Medical Imaging, University of Toronto, Toronto, Ontario M4N 3M5, Canada

R. S. Hinks

Sunnybrook Health Science Centre, and Department of Medical Biophysics, University of Toronto, Toronto, Ontario M4N 3M5, Canada

J. W. Hunt

Ontario Cancer Institute/Princess Margaret Hospital, and Department of Medical Biophysics,

University of Toronto, Toronto, Ontario M5G 2M9, Canada

B. C. Wilson

Ontario Cancer Institute/Princess Margaret Hospital, and Department of Medical Biophysics, University of Toronto, and Ontario Laser and Lightwave Research Centre, Toronto, Ontario M5G 2M9, Canada

A. C. Easty

The Toronto Hospital, and Institute of Biomedical Engineering, University of Toronto, Toronto, Ontario M5G 2C4, Canada

M. J. Bronskill

Sunnybrook Health Science Centre, and Departments of Medical Biophysics and Medical Imaging, University of Toronto, Toronto, Ontario M4N 3M5, Canada

W. Kucharczyk

The Toronto Hospital, and Department of Medical Imaging, University of Toronto, Toronto, Ontario M5S 1A8, Canada

M. D. Sherar

Ontario Cancer Institute/Princess Margaret Hospital, and Department of Medical Biophysics, University of Toronto, Toronto, Ontario M5G 2M9, Canada

R. M. Henkelman

Sunnybrook Health Science Centre, and Departments of Medical Biophysics and Medical Imaging,

University of Toronto, Toronto, Ontario M4N 3M5, Canada

(Received 15 April 1996, accepted for publication 5 November 1996)

Changes in magnetic resonance (MR) signals during interstitial microwave heating are reported, and correlated with simultaneously acquired temperature readings from three fiber-optic probes implanted in a polyacrylamide gel phantom. The heating by a MR-compatible microwave antenna did not interfere with simultaneous MR image data acquisition. MR phase-difference images were obtained using a fast two-dimensional-gradient echo sequence. From these images the temperaturesensitive resonant frequency of the ${ }^{1} \mathrm{H}$ nuclei was found to decrease approximately by $0.008 \mathrm{ppm} /$ ${ }^{\circ} \mathrm{C}$. The method and results presented here demonstrate that noninvasive MR-temperature imaging can be performed simultaneously with interstitial microwave thermal treatment. (C) 1997 American Association of Physicists in Medicine. [S0094-2405(97)00502-6]

Key words: MRI, microwave heating, temperature monitoring, phase-shift imaging 


\section{INTRODUCTION}

The use of magnetic resonance imaging (MRI) to monitor and guide minimally invasive therapeutic interventions is currently under active investigation. ${ }^{1-4}$ The noninvasive nature of MRI coupled with its ability to produce threedimensional (3D) anatomical images in any orientation with good spatial resolution makes it a potentially attractive modality for this purpose. Recent work in this area has focused on thermal therapy, an experimental treatment of malignancies and other diseases. The goal of thermal therapy is to raise the diseased tissue temperature to a sufficiently high level for a sufficiently long time to cause localized cell death while ensuring that the corresponding "thermal dose" to surrounding healthy tissues does not produce damage. ${ }^{5} \mathrm{An}$ other active and related research area is thermal dose monitoring during thermal therapy. ${ }^{5}$ This is because the cytotoxic effect of heat is strongly dependent on the time-temperature history of a given tissue volume. ${ }^{6}$

Whether the therapy involves high temperatures and short times as in thermal coagulation therapy $\left(\sim 60^{\circ} \mathrm{C}\right.$, several minutes), or low temperatures and long times as in conventional hyperthermia $\left(\sim 43^{\circ} \mathrm{C}\right.$, tens of minutes), it is desirable to characterize the spatial and temporal tissue temperature distributions during the therapy to predict the extent of the resultant thermal damage to tissue. If the temperature distributions are obtained and updated during the thermal treatment, they may be used to guide the treatment in real time in order to match the thermal damage zone to the intended target volume.

Traditionally, temperature monitoring during thermal therapy has been achieved using interstitial thermocouples, thermistors, or fiber-optic probes. ${ }^{7-9}$ Unfortunately, these measurements, although accurate, possess two serious shortcomings: they are invasive, and they yield only a spatially localized and thus a sparse data set which must be interpolated or extrapolated to predict temperatures throughout the treatment volume. Accurate 3D transient thermal modeling is thus a formidable task. In addition to the usual complexity of solving the heat conduction equation in an irregularly shaped volume, the effects of tissue heterogeneity, blood flow, and possible changes in tissue properties during the treatment must be included. Even if a suitable model is developed, the accuracy of the data and the length of time required for computation makes real-time temperature guidance difficult. Clearly, a noninvasive volumetric imaging technique that can "map" temperature directly rather than calculate it from point measurements is desirable. MRI is currently being investigated for this purpose.

The use of MRI to monitor temperature changes in vivo was first suggested over a decade ago. ${ }^{10,11}$ Since then, the temperature sensitivity of various MR parameters has been investigated. ${ }^{12-23}$ These and other studies have used MR imaging sequences optimized to measure the temperatureinduced changes in the ${ }^{1} \mathrm{H}$ nucleus relaxation times ( $\mathrm{T} 1$ and T2) and resonance frequency shift, as well as molecular diffusion. Unfortunately, standard diagnostic imaging sequences can require several minutes to visualize the desired changes, when near real-time performance is desirable. Some recent in vivo and ex vivo animal studies have investigated the utility of a number of MRI pulse sequences for temperature monitoring. ${ }^{18,24}$

In this paper, we report on the use of a commercially available 2D fast spoiled gradient-echo sequence (SPGR) to monitor temperature changes during interstitial microwave heating of a polyacrylamide gel. We have employed the ${ }^{1} \mathrm{H}$ proton resonance-shift method for correlating MR signal changes with temperature changes in the heated gel. Briefly, the NMR frequency of pure water protons decreases by approximately $0.01 \mathrm{ppm} /{ }^{\circ} \mathrm{C}$ as a result of the exchange between hydrogen-bonded and unbonded water, and stretching and bending of hydrogen bonds among water molecules. ${ }^{25,26}$ The thermally induced changes in the electronic configuration of the hydrogen bonds result in increased average magnetic shielding of the protons, which decrease the local magnetic field experienced by the protons and, consequently, their NMR frequency. The proton resonance frequency shift is found to be a linear function of temperature for water in the liquid state, ${ }^{25}$

$$
|\Delta f|=\left(0.01 \mathrm{ppm} /{ }^{\circ} \mathrm{C}\right) f_{0} \Delta T \text {. }
$$

At a field strength of $1.5 \mathrm{~T}$ (used in many clinical MRI scanners), the Larmor frequency, $f_{0}$, of water protons is 64 $\mathrm{MHz}$, yielding a thermal coefficient, $\Delta f / \Delta T$, of approximately $-0.64 \mathrm{~Hz} /{ }^{\circ} \mathrm{C}$.

Shifts in the resonant frequency of water protons can be measured using a gradient-echo MR imaging pulse sequence which detects changes in the phase of the proton's NMR signal. After waiting an echo time (TE) of several milliseconds, the measured phase difference in the NMR signal of pure water, $\Delta \phi$, from a temperature change of $\Delta T$ is

$$
|\Delta \phi|=\left(0.64 \mathrm{~Hz} /{ }^{\circ} \mathrm{C}\right) \mathrm{TE} \Delta T .
$$

Since the MR imaging sequence spatially resolves the MR signals, the calculated phase changes are also spatially resolved. Consequently, the above equation can be inverted to derive a temperature map of the imaging volume, $\Delta T(\mathbf{r}) .{ }^{19-23}$

Several noninvasive or minimally invasive ways to administer heat into a target volume have been reported. Many use electromagnetic energy, such as (i) visible to nearinfrared light via an interstitial optical fiber, ${ }^{27,28}$ (ii) microwave via an interstitial antenna ${ }^{29}$ or external applicator arrays,${ }^{30}$ and (iii) radio frequency via interstitial or external applicators. ${ }^{14,31}$ It is also possible to deliver thermal energy using ultrasound applicators, both external ${ }^{32}$ and interstitial. ${ }^{33,34}$ Each method has its advantages and disadvantages with respect to many considerations, such as electromagnetic interference with MR imaging and lesion size. 


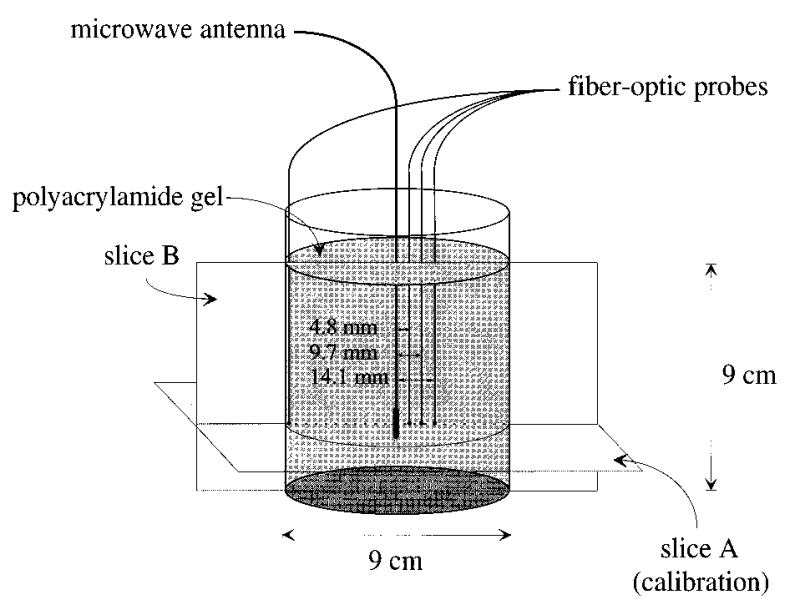

FIG. 1. Geometry of the polyacrylamide gel phantom. Shown are the positions of the microwave antenna and fiber-optic temperature probes. MR images were acquired from two perpendicular slices (slices A and B). Region-of-interest analysis of slice A provided a calibration with which to derive temperature maps from MR images of slice $\mathrm{B}$.

Recently, we and others have found that an interstitial microwave antenna might be beneficial for the treatment of deep-seated lesions because it produces radiation that penetrates well into tissue, providing a large (on the order of centimeters), more evenly heated treatment volume. ${ }^{35,36}$

The utility of MRI monitoring during interstitial microwave thermal therapy has not been investigated in detail, primarily because of the metallic nature of the microwave antenna and its possible MR incompatibility. Conventional antenna designs generally distort the homogeneous magnetic field, interfere with the radio frequency fields, and establish eddy currents during gradient-field switchings. Attempts to circumvent these difficulties have been reported, including the construction of surface microwave applicators without ferromagnetic components that are used with the energy switched off during the MRI signal acquisition to minimize possible noise interference. ${ }^{14,31}$ Our investigation of interstitial microwave heating has necessitated the development of MRI-compatible antennas and a choice of microwave operating frequency that would allow MR data acquisition with the microwave power on. Recently, initial success has been reported by ourselves and other groups in monitoring interstitial microwave-induced heating patterns in phantoms ${ }^{35}$ and lesion formation ex vivo. ${ }^{36}$ The objectives of this study are threefold: (1) to verify that MRI can be performed simultaneously with interstitial microwave heating; (2) to correlate the MR phase changes with fiber-optic temperature measurements; and (3) to compare qualitatively the MRI-derived 3D temperature maps with results predicted from thermal modeling.

\section{MATERIALS AND METHODS}

A polyacrylamide (Sigma Chemicals, St. Louis, MO) gel phantom (shown in Fig. 1) was specifically prepared to mimic the conductive and dielectric properties of human muscle in the microwave $(200-2500 \mathrm{MHz})$ region of the electromagnetic spectrum. ${ }^{37}$ Prior to solidification, approximately 0.51 of gel was poured into a cylindrical Pyrex ${ }^{\mathrm{TM}}$ beaker $9 \mathrm{~cm}$ in diameter. Five Teflon ${ }^{\mathrm{TM}}$ catheters were positioned in the gel, parallel to the cylindrical axis during solidification: one 1.9-mm-o.d. (0.35 mm wall thickness) catheter to accommodate the microwave antenna, and four 1.0mm-o.d. (0.15 mm wall thickness) catheters for the fiberoptic temperature probes. The fiber-optic probes were placed in the catheters at radial distances from the microwave antenna of approximately 5, 10, 15, and $43 \mathrm{~mm}$.

Prior to the imaging experiments, some relevant temperature-sensitive NMR properties of the phantom were measured to determine their contributions to temperature contrast. NMR spectroscopy was performed on a $1.5 \mathrm{~T}$ small-bore spectrometer to measure the proton resonance frequency shift of the polyacrylamide gel as a function of temperature, over the range of $20-55^{\circ} \mathrm{C}$. The resonance frequency decreased with temperature by $0.0091 \pm 0.0007$ [standard deviation (s.d.) $] \mathrm{ppm} /{ }^{\circ} \mathrm{C}$, a thermal coefficient that is slightly smaller than the value of $0.01 \mathrm{ppm} /{ }^{\circ} \mathrm{C}$, usually stated for pure water. ${ }^{19-23}$

NMR relaxometry ${ }^{38}$ was also performed on the spectrometer to gain insight into the nuclear relaxation times of the gel as a function of temperature. The results indicated primarily a single-component longitudinal (T1) relaxation time of approximately $1010 \mathrm{~ms}$ (incorporating 90\% of the protons) at $20^{\circ} \mathrm{C}$, which did not vary significantly with temperature over the range of $20-55^{\circ} \mathrm{C}$. The transverse (T2) magnetization was found to be more of a multicomponent nature, with the primary component at $720 \mathrm{~ms}$ (incorporating $70 \%$ of the protons) at $20{ }^{\circ} \mathrm{C}$. The relaxation time of this component decreased with increasing sample temperature, dropping approximately linearly to $215 \mathrm{~ms}$ at $55^{\circ} \mathrm{C}$ (for comparison, T1 and T2 times of human muscle at $37^{\circ} \mathrm{C}$ are 870 and $47 \mathrm{~ms}$, respectively ${ }^{38}$ ). These relaxometric results indicate that $\mathrm{T} 1$ temperature sensitivity was not the preferred mechanism to observe temperature changes through $\mathrm{MR}$ imaging for this particular phantom material.

The microwave antenna was specifically designed to be MR compatible, and was provided through the courtesy of Dornier Medical Systems Incorporated (Kennesaw, GA). The antenna had a helical emitter at its tip of $26 \mathrm{~mm}$ length ( $1 \mathrm{~mm}$ diam) and was driven by a frequency generator in series with an amplifier operating at $915 \mathrm{MHz}$, a frequency commonly used for microwave hyperthermia. The power delivered to the antenna tip was $11.5 \mathrm{~W}$. Due to its MR incompatibility, the microwave supply was positioned outside the MR scanner room and the antenna was connected to it via a microwave coaxial cable with a common ground at the junction box of the MR room. This arrangement was found to produce less image noise than feeding the cable directly through a waveguide opening in the wall.

Direct temperature measurements were made at three collinear points within $15 \mathrm{~mm}$ of the microwave antenna, and one at the wall of the gel-filled beaker with fluorescent fiberoptic probes (Luxtron Corp., Santa Clara, CA). The fiberoptic probes did not cause electromagnetic interference with the MR measurements and were assumed to have a negli- 
gible effect on the thermal distribution due to their small size and low thermal conductivity. The probe tips were positioned at the level of the midpoint of the helical emitter (Fig. $1)$. The thermometry system was calibrated at one point $(T$ $=29.77^{\circ} \mathrm{C}$ ) with a gallium temperature standard; the absolute accuracy of the reported fiber-optic measurements is $\pm 0.3{ }^{\circ} \mathrm{C}$ (manufacture's specification), over the range of temperatures studied. When placed in a common thermal environment, we determined the precision of each of the four fiber-optic probes to be $\leqslant \pm 0.1^{\circ} \mathrm{C}$ (s.d.), and a probe-toprobe variation to be $\leqslant \pm 0.2{ }^{\circ} \mathrm{C}$ (s.d.), which is consistent with the reported accuracy of the temperature measurements. The Luxtron unit took approximately $2 \mathrm{~s}$ to make a temperature measurement (for all four probes). Measurements were recorded every $20.0 \mathrm{~s}$, which was adequate to monitor the temperature profiles. These temperature readings were then correlated with the MR phase changes induced by the microwave heating.

For the purpose of subsequent thermal modeling, we estimated the 3D emitted power pattern (specific absorption rate, SAR) of the microwave antenna from a series of $2 \mathrm{D}$ measurements. These were performed in a stack of $2.5-\mathrm{mm}-$ thick polyacrylamide gel slabs using a thermographic IR camera (Inframetrics Model 522, Billerica, MA). The camera produces a $512 \times 512$ pixel image with a temperature resolution of $0.1{ }^{\circ} \mathrm{C}$. To minimize the effects of heat conduction during the measurement, ${ }^{37}$ high power $(10 \mathrm{~W})$ was applied for a short time $(20 \mathrm{~s})$ to the antenna. A thermal image was recorded in four consecutive planes parallel to the antenna axis at distances of $2.5 \mathrm{~mm}$ apart, with one measurement after each heating pulse. The time from turning off the power, peeling off the appropriate gel slab(s), to recording the image, was typically $5 \mathrm{~s}$. The maximum temperature rise was $6{ }^{\circ} \mathrm{C}$. The SAR was calculated according to

$$
\mathrm{SAR}=\rho c \frac{\Delta T}{\Delta t},
$$

where $\rho c$ is the volumetric specific heat, $\Delta T$ is the temperature rise, and $\Delta t$ is the heating time. This assumes a linear temperature rise with time, which is a reasonable assumption if thermal conduction is minimal. This is expected to be the case for this measurement, since the heating time is only 20 s. ${ }^{37,39}$ The effects of thermal conduction on the accuracy of the infrared thermographic SAR measurements of interstitial microwave antennas are currently under investigation. ${ }^{39}$

MR imaging was performed on a standard $1.5 \mathrm{~T}$ clinical whole-body MRI system (Signa; GE Medical Systems, Milwaukee, WI). A standard SPGR sequence was used with a 5 in. surface coil. The imaging parameters were: tip angle $=30^{\circ}$, echo time $(\mathrm{TE})=10.1 \mathrm{~ms}$, repetition time $(\mathrm{TR})=31.8$ $\mathrm{ms}$, number of signal averages $(\mathrm{NSA})=2$, slice thickness $=5$ $\mathrm{mm}, \mathrm{FOV}=9 \mathrm{~cm} \times 9 \mathrm{~cm}, 256 \times 256$ image matrix, and an imaging time of $16.3 \mathrm{~s}$. From two separate microwave heating experiments in the same gel, MR images of two perpendicular slices (slices A and B in Fig. 1) were obtained. Approximately $100 \mathrm{MR}$ images were collected from each experiment, each lasting approximately $30 \mathrm{~min}$.
For each experiment, after obtaining at least ten MR images (to define an initial average reference image), the microwave power was turned on to deliver $11.5 \mathrm{~W}$ to the gel. After 4 min of heating, the microwave power was turned off; MR imaging then continued through the cooling phase of the gel. At least $40 \mathrm{~min}$ elapsed between the end of the first experiment (acquisition of slice A) and the start of the second experiment (slice B), to ensure that the gel had cooled to room temperature. No irreversible changes were visually observed in the gel as a result of these repeated heating and cooling cycles.

The first step in correlating the MR phase changes with the fiber-optic temperature readings was to bring the two sets of discrete measurements into temporal correspondence. The fiber-optic temperature measurements were interpolated in time to correspond with the time when the center of the raw data matrix ( $k$ space) of the MR image was filled.

Phase difference images were calculated from each complex-valued digital MR image. The first ten images, acquired before microwave heating, were averaged (for noise reduction) to form a single reference phase image which was subtracted from each subsequent MR phase image.

It was found that the phase-difference measurements from the MR images required a correction for temporal fluctuations or instabilities either in the static magnetic field, $B_{0}$, and/or the standard reference frequency (as either would effect the phase), although the exact source of the temporal fluctuations was not determined. This correction, of the order of $\sim \leqslant 0.2 \mathrm{ppm}$ (over $26 \mathrm{~min}$ ), was performed by subtracting the phase of a small region in the FOV which experienced no temperature change from the entire MR phase image to remove any nonthermal effects from the phase images. In this study, we used a small regions-of-interest (ROI) on the periphery of the gel phantom (near the end fiber optic adjacent to the glass wall-see Fig. 1) which did not deviate by more than $1.0^{\circ} \mathrm{C}$ over the entire microwave heating and cooling period. While this method was found to be sufficient for our purposes of correlating MR phase changes with fiber-optic temperatures, an alternative approach would be to use one or more external reference phantoms (that are not heated, but are within the imaging FOV) for the sole purpose of this necessary temporal phase correction.

Three annular ROIs were placed concentrically with the position of the microwave antenna within which MR phase differences were computed and correlated with the three fiber-optic temperatures (see Fig. 2). This provided a way to relate the MR phase changes to temperature and to predict the 3D temperature distribution from the second heating experiment (slice B). The latter results enabled a direct comparison of the MRI-derived temperature with thermal modeling predictions, using the measured SAR pattern of the microwave antenna.

The purpose of the thermal modeling was to verify that the temperatures calculated from MR phase change images (slice B) were consistent with the predicted temperature patterns. To calculate the temperature distribution, the 3D heat conduction equation, 


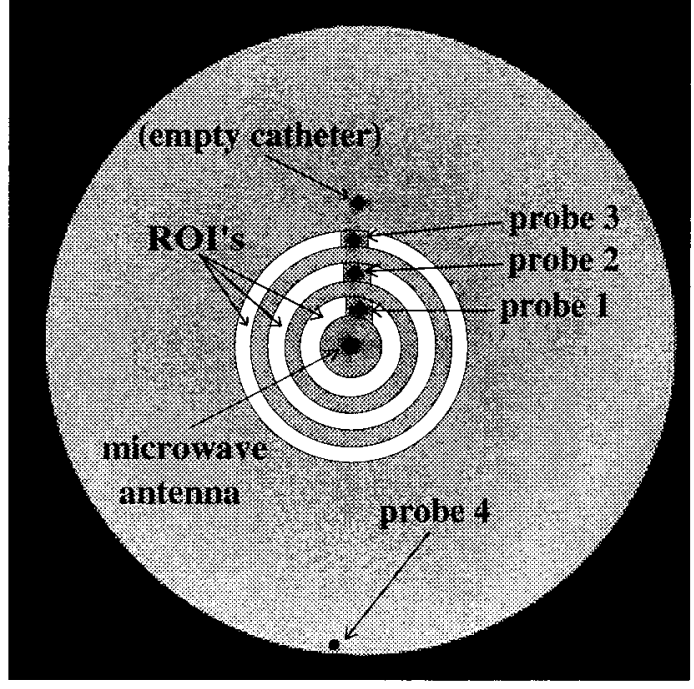

FIG. 2. Magnitude MR image of slice A (prior to heating) indicating the three regions-of-interest used in temperature calibration. The top-most hole is a catheter that did not contain a fiber-optic temperature probe. Relevant MR imaging parameters: tip angle $=30^{\circ}, \mathrm{TE}=10.1 \mathrm{~ms}, \mathrm{TR}=31.8 \mathrm{~ms}$, $\mathrm{NSA}=2$, slice thickness $=5 \mathrm{~mm}, \mathrm{FOV}=9 \mathrm{~cm} \times 9 \mathrm{~cm}, 256 \times 256$ image matrix.

$$
\rho c \frac{\partial T}{\partial t}=k\left(\frac{\partial^{2} T}{\partial x^{2}}+\frac{\partial^{2} T}{\partial y^{2}}+\frac{\partial^{2} T}{\partial z^{2}}\right)+P .
$$

was solved using a finite difference time domain alternatedirection-implicit algorithm. ${ }^{40}$ The thermal conductivity value used was $k=0.00395 \mathrm{~W} \mathrm{~cm}^{-1} \mathrm{~K}^{-1}$, and the volumetric specific heat was $\rho c=4.077 \mathrm{~J} \mathrm{~cm}^{-3} \mathrm{~K}^{-1}, 37$ The power term, $P(x, y, z)$, was determined by the experimentally measured SAR pattern. The computational grid was $9 \times 10 \times 9$ $\mathrm{cm}$. The spatial increment in all directions was $2.5 \mathrm{~mm}$; to ensure solution stability, the temporal step size was limited to $0.1 \mathrm{~s}$. A constant ambient temperature of $20{ }^{\circ} \mathrm{C}$ was imposed on all boundaries.

\section{RESULTS}

Figure 3 shows the central plane SAR pattern of the antenna. The resultant tear-drop SAR pattern is typical of helical emitters, where the heating area is located near the tip of the antenna, although the maximum SAR is slightly closer to the center of the emitter than the helical antenna tested by Ryan. ${ }^{41}$ The $50 \%$ SAR contour is approximately $8 \mathrm{~mm}$ in width in this plane. From examination of other planes, the $50 \%$ contour was seen to be cylindrically symmetric about the antenna axis.

Representative MR phase images of microwave heating and subsequent cooling of the gel phantom (in slice A) are illustrated in Fig. 4. Figure 4(a) is one of ten baseline MR images that were used to compute a reference phase image which was subtracted from all of the subsequent phase images. With the microwave power on, the phase images clearly show a well-defined, slightly oval region of phase enhancement centered about the microwave antenna [Figs. $4(\mathrm{~b})-4(\mathrm{~g})]$. After the microwave power is turned off, the

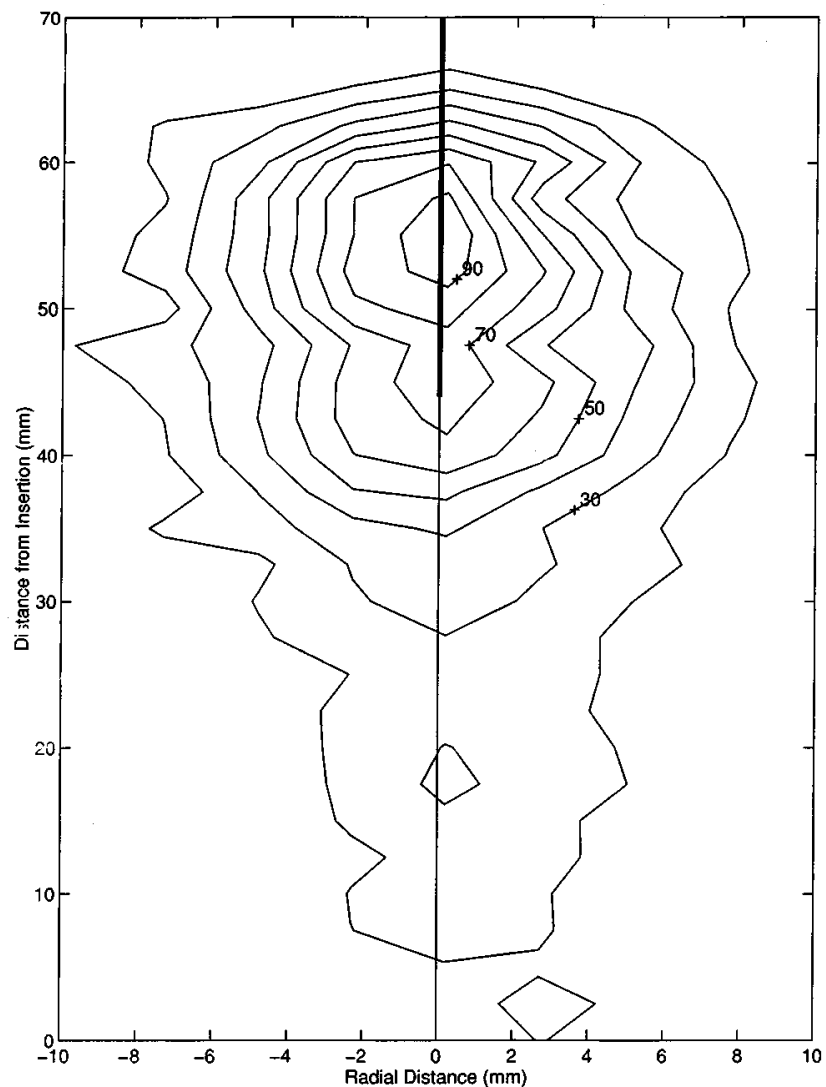

FIG. 3. SAR heating pattern of the helical Dornier microwave antenna. Infrared temperature measurements were recorded in successive $2.5-\mathrm{mm}$ slices of a polyacrylamide gel. Shown is the heating pattern in the central plane with percentage contours normalized to the maximum SAR. The origin of the $y$ axis denotes the insertion point of the antenna into the gel, and $70 \mathrm{~mm}$ corresponds to the tip of the antenna. The helical emitting element is displayed in bold.

edges of the region becomes blurred, most likely due to the diffusion of heat through the gel [Figs. 4(h)-4(j)].

Annular ROI analysis of the phase images was performed as described above. The results are presented in Fig. 5, and show excellent agreement between the fiber-optic probe temperatures and the MR phase changes over the entire temporal range of the experiment, at each of the three spatial probe locations. A calibration plot of the same ROI data is presented in Fig. 6, which reveals a near one-to-one relationship between MR phase change and fiber-optic temperature, which we perceive as a true measure of the actual local gel temperature (reported accuracy of $\pm 0.3{ }^{\circ} \mathrm{C}$ s.d.). The actual pixels representing the microwave antenna (within the central black region-see Fig. 4) were chosen to minimize the least-squares sum of a linear fit between the MR phase changes and fiber-optic temperatures, within a pixel resolution $(0.35 \mathrm{~mm})$. We found that a shift of this position by more than two pixels $(0.7 \mathrm{~mm})$ significantly affected the features of the calibration plot, and Fig. 6 shows the best fit obtained, namely

$$
|\Delta \phi|=\left(-38.0 \pm 0.2^{\circ}\right)+\left(1.94 \pm 0.01^{\circ} /{ }^{\circ} \mathrm{C}\right) \Delta T,
$$



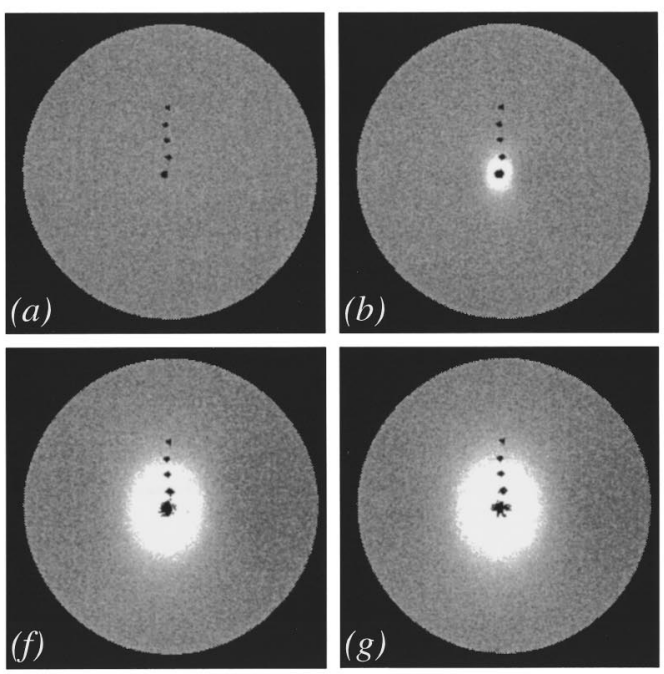
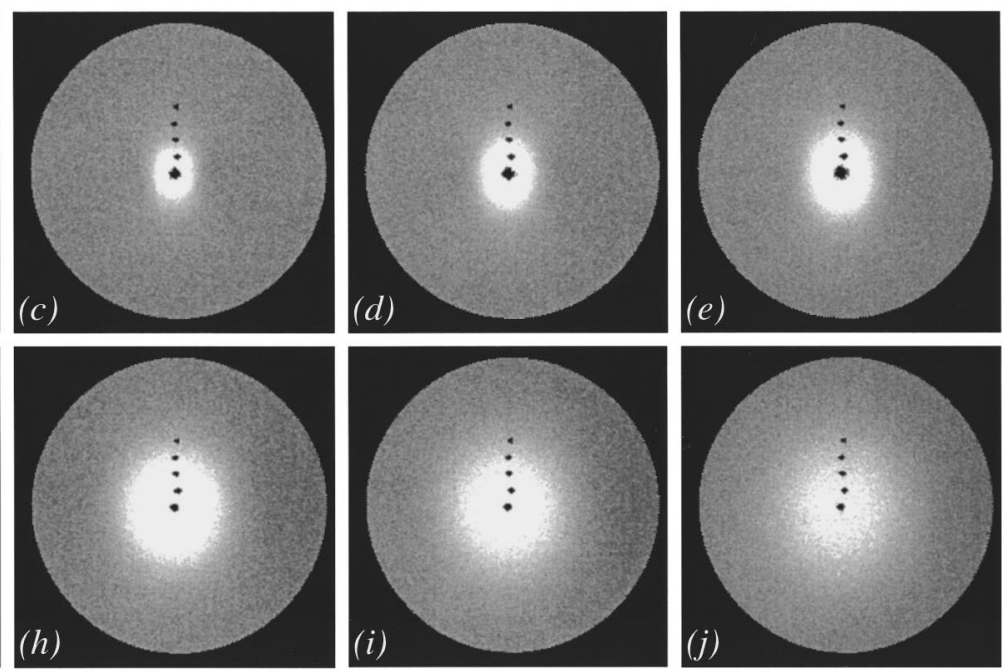

FIG. 4. MR phase images of slice A during microwave heating and cooling. The images were acquired at approximate times: (a) $-196 \mathrm{~s}$, (b) $0 \mathrm{~s}$, (c) $33 \mathrm{~s}$, (d) $82 \mathrm{~s}$, (e) $130 \mathrm{~s}$, (f) $196 \mathrm{~s}$, (g) $261 \mathrm{~s}$, (h) $359 \mathrm{~s}$, (i) $538 \mathrm{~s}$, and (j) $685 \mathrm{~s}$ relative to when the microwave power was turned on $(t=0 \mathrm{~s})$. The microwave power supply was turned off at $t=261 \mathrm{~s}$. The background image, of which (a) is one scan, has been subtracted from all subsequent images (b)-(j). Images (b)-(g) depict the effect of microwave heating, where images (h)-(j) describe cooling of the polyacrylamide gel. All phase images were leveled to $175^{\circ}$ and windowed to $50^{\circ}$ to demonstrate the thermal lesion and presence of background noise.

where $\Delta T=T-19.4^{\circ} \mathrm{C}$ and the above uncertainties are the standard deviations from the linear fit. The TE used in the MR imaging pulse sequence was $10.1 \mathrm{~ms}$, therefore the resonant frequency of the ${ }^{1} \mathrm{H}$ nuclei decreased by approximately $0.0083 \mathrm{ppm} /{ }^{\circ} \mathrm{C}$. This result is slightly lower than the spectroscopically derived result of $0.0091 \mathrm{ppm} /{ }^{\circ} \mathrm{C}$ for the same gel.

Once the data for the calibration had been obtained (slice A in Fig. 1), MR imaging of a perpendicular slice (slice B in Fig. 1) was undertaken, under identical microwave heating conditions. The above calibration was used to derive thermal maps from the phase images. Representative MR-derived thermal maps of slice B are presented in Fig. 7. Note the

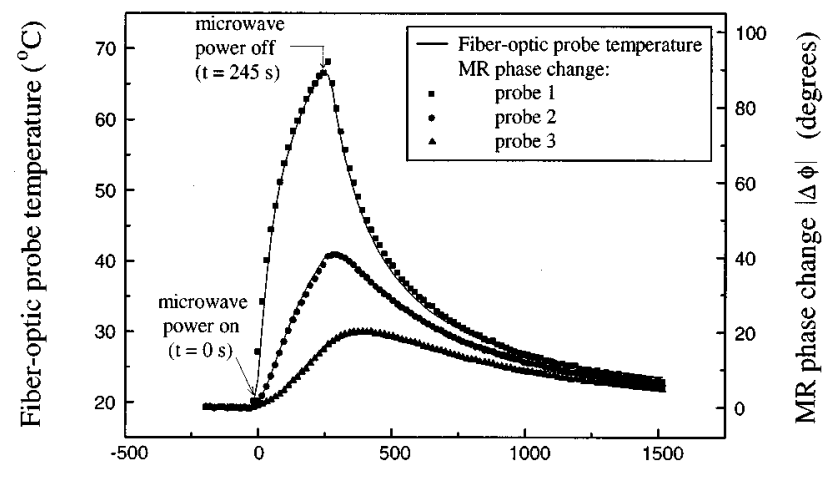

Time (seconds)

FIG. 5. Fiber-optic probe temperature and MR phase change as a function of time (slice A). Shown are fiber-optic and MR phase change measurements for each of the three fiber-optic probes and regions-of-interest, as indicated in Fig. 2. The MR phase measurements (right vertical axis) were scaled to the fiber-optic temperatures (left vertical axis) using the calibration curve in Fig. 6. tear-drop thermal pattern emerging with time, centered about the microwave antenna, similar to its SAR pattern. Again, the pattern in Figs. 7(h)-7(j) disperses after the microwave power is turned off.

Temperature distributions calculated from Eq. (4), and corresponding to the same times as in Fig. 7 (excluding the two background images for times $t \leqslant 0 \mathrm{~s}$ ), are shown for comparison in Fig. 8. The similar shape of the thermal patterns in Figs. 7 and 8 demonstrates general agreement between temperature changes measured via MR phase change and those predicted theoretically.

\section{DISCUSSION AND CONCLUSIONS}

The results presented here show that the temperature distributions in interstitial microwave thermotherapy can be ac-

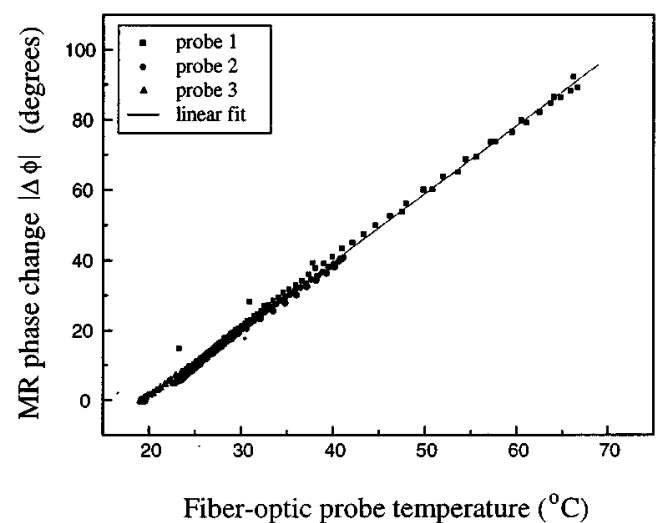

FIG. 6. MR phase change vs fiber-optic probe temperature (slice A). The phase changes from all three ROIs are plotted against their corresponding fiber-optic probe temperatures from the data presented in Fig. 5, for both heating and cooling cycles. A linear fit yielded a slope of $|\Delta \phi| / \Delta T=1.94$ $\pm 0.01 \%{ }^{\circ} \mathrm{C}$. 

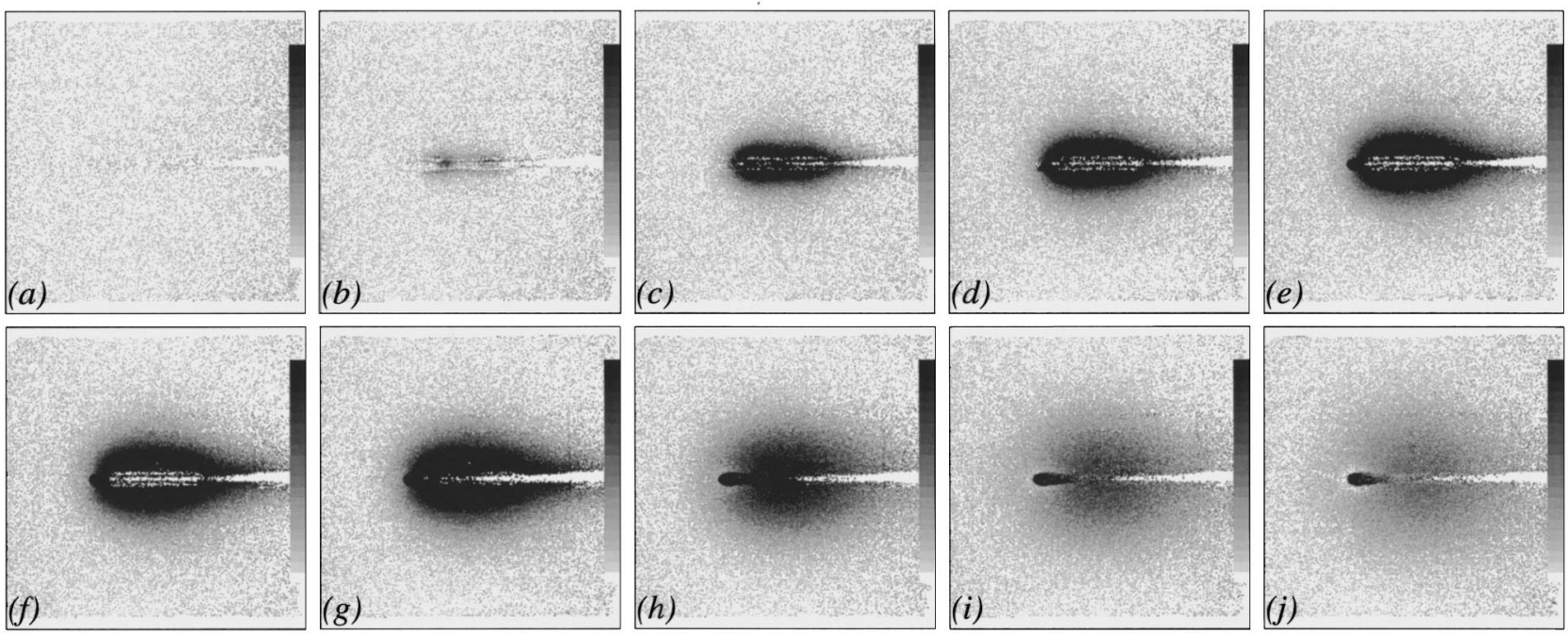

FIG. 7. MRI-derived temperature images of slice B during microwave heating and cooling. The images were acquired at approximate times: (a) -179 s, (b) $0 \mathrm{~s}$, (c) $49 \mathrm{~s}$, (d) $98 \mathrm{~s}$, (e) $147 \mathrm{~s}$, (f) $196 \mathrm{~s}$, (g) $245 \mathrm{~s}$, (h) $408 \mathrm{~s}$, (i) $571 \mathrm{~s}$, and (j) $734 \mathrm{~s}$ relative to when the microwave power was turned on $(t=0 \mathrm{~s})$. The microwave power supply was turned off at $t=245 \mathrm{~s}$. The background image, of which (a) is one scan, has been subtracted from all subsequent images (b) -(j). Images (b) $-(\mathrm{g})$ describe the effect of microwave heating, whereas images (h) $-(\mathrm{j})$ describe cooling of the polyacrylamide gel. All temperature images were leveled to $29.5^{\circ} \mathrm{C}$ and windowed to $20.0^{\circ} \mathrm{C}$. The contrast was reversed for display purposes. The scale shown on the images increments in steps of $1.0{ }^{\circ} \mathrm{C}$ (from 19.5 to $39.5^{\circ} \mathrm{C}$ ).

curately monitored in a near-real-time fashion using MRI with minimal distortion of the MR image. The use of microwaves may offer specific advantages over other heat-delivery techniques. For example, the increased penetration of microwaves compared to near-infrared laser energy may result in larger volumes of significant temperature elevation and hence in larger volumes of coagulated tissue. Since this treat- ment modality can also be monitored and guided in nearreal-time via MRI it has significant therapeutic potential.

One interesting observation from the MR thermometry concerns the conduction of heat within the gel, and more generally in tissue. It is observed that the three fiber-optic probe temperatures (and phase changes) peaked at different points in time. This is most easily seen in the data of Fig. 5.

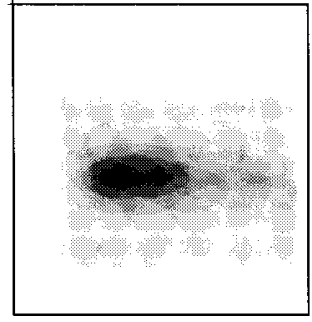

(a)

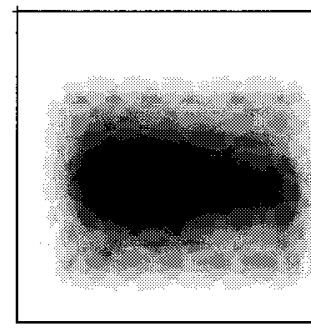

(e)

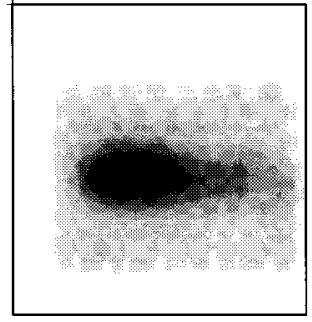

(b)

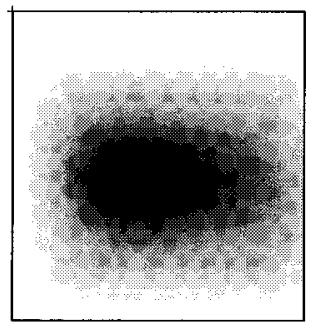

(f)

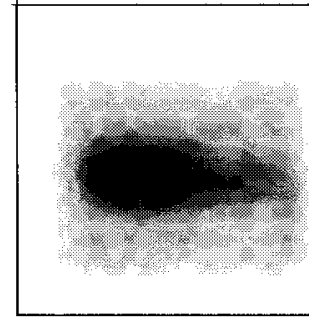

(c)

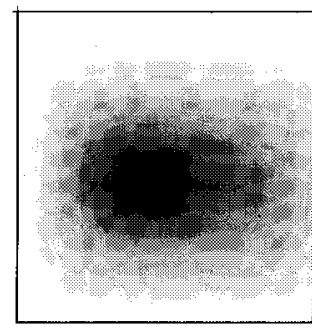

$(\mathrm{g})$

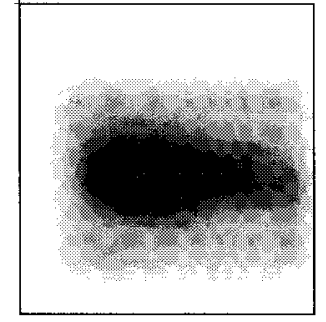

(d)

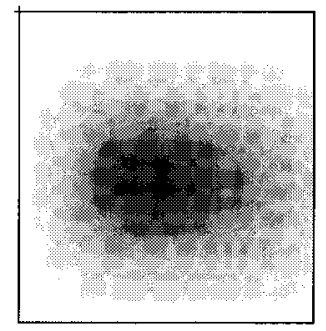

(h)

FIG. 8. Temperature patterns predicted by thermal modeling for the same times as the MRI-derived temperature images shown in Fig. 7 [excluding the times $t \leqslant 0$, so that (a) is to be compared with $7(\mathrm{c}), 7(\mathrm{~b})$ with $7(\mathrm{~d})$, etc]. The SAR was scaled by matching the temperature predicted by the model to that recorded by probe 1 in the calibration curve (Fig. 5), at the time the microwave power was switched off. Black corresponds to temperatures $\geqslant 20{ }^{\circ} \mathrm{C}$ above background (white), to enable comparison with the MR-derived thermal maps (Fig. 7). 
The temperature of the first fiber-optic probe (located at 4.8 $\mathrm{mm}$ from the microwave antenna) peaked at approximately $265 \mathrm{~s}$, which is within a few seconds of the microwave power being turned off. The temperature of the second $(9.7$ $\mathrm{mm})$ and third $(14.1 \mathrm{~mm})$ fiber-optic probes peaked at approximately $294 \mathrm{~s}$ (33 s after power was off) and $391 \mathrm{~s} \mathrm{(130}$ $\mathrm{s}$ after power was off), respectively. This is a consequence of the thermal conduction process (thermal conduction times scale as the square of the distance divided by the medium's thermal diffusivity, hence a distant point will be much delayed in experiencing the change in the thermal status at the position of the microwave antenna-in this case, the change being the termination of microwave heating). This may have clinical significance when the methodology is applied in vivo, because tissue more than a few millimeters away from the thermal source may continue to heat up even after the microwave power has been turned off. This is particularly relevant to thermal dosimetry of surrounding healthy tissues. However, the effects of blood flow may mask this observation.

We believe that more work needs to be done before MRI monitoring of microwave thermal therapy is used in a clinical environment. The effects of patient and organ motion, the effects of blood flow, and the inhomogeneity of real tissues will influence both the thermal distributions and the quality and appearance of the MR images. In addition, molecular alterations during thermal treatment in tissues elevated to therapeutic temperatures will likely result in MRI signal changes with a complex dependence on both temperature and molecular changes. This is a more complicated situation than examined in the present study, where effects of temperature and molecular structure were fairly well decoupled, as there did not appear to be any irreversible molecular changes to the gel.

This paper demonstrates the feasibility of quantitative MR monitoring of microwave-administered interstitial thermal therapy. Simultaneous heating and MR thermometry were demonstrated with a $2 \mathrm{D}$ fast gradient echo technique.

\section{ACKNOWLEDGMENTS}

R.D.P. is a recipient of a Medical Research Council of Canada doctoral scholarship. M.C.K. is a recipient of a University of Toronto Open Doctoral Scholarship. Thanks are due to W. Taylor, K. Grant, and A. Worthington for skillful technical assistance. This work is part of the image guided minimally invasive therapy (IGMIT) project at the University of Toronto. IGMIT is a partnership of Sunnybrook Health Science Centre, The Toronto Hospital, General Electric Medical Systems of Canada, and ISG Technologies, which receives significant funding from Technology Ontario as well as from the partners. Additional support from Dornier Medical Systems Incorporated for providing the microwave antenna and power source, and from the National Cancer Institute of Canada (I.A.V. and M.D.S.) are also gratefully acknowledged.

${ }^{a}$ Correspondence address: Department of Medical Physics, Princess Margaret Hospital, 610 University Avenue, Toronto, Ontario M5G 2M9,
Canada; tel: (416)946-2000; fax: (416)946-6566; electronic-mail: vitkin@clinphys.pmh.toronto.on.ca

${ }^{1}$ H. E. Cline, J. F. Schenck, R. D. Watkins, K. Hynynen, and F. A. Jolesz, "Magnetic resonance-guided thermal surgery," Magn. Reson. Med. 30, 98-106 (1993).

${ }^{2}$ J. F. Schenck et al., "Superconducting open-configuration MR imaging system for image-guided therapy," Radiology 195, 805-814 (1995).

${ }^{3}$ R. Lufkin, "Interventional MR imaging," Radiology 197, 16-18 (1995).

${ }^{4}$ S. G. Silverman et al., "Interactive MR-guided biopsy in an openconfiguration MR imaging system," Radiology 197, 175-181 (1995).

${ }^{5}$ Hyperthermia Treatment Planning, AAPM Report No. 27 (American Institute of Physics, New York, 1989).

${ }^{6}$ J. Pierce and S. Thomsen, "Rate process analysis of thermal damage," in Optical-Thermal Response of Laser-Irradiated Tissue, edited by A. J. Welch and M. J. C. van Gemert (Plenum, New York, 1995), pp. 561606.

${ }^{7}$ N. Daikuzono, M. Suzuki, and S. N. Joffe, "Computer controlled contact Nd:YAG laser system for interstitial laser hyperthermia," Lasers Surg. Med. 7, 101-110 (1987).

${ }^{8}$ S. T. Clegg and R. B. Roemer, "Towards the estimation of threedimensional temperature fields from noisy temperature measurements during hyperthermia,' Int. J. Hyperthermia 5, 467-473 (1989).

${ }^{9}$ D. R. Wyman, C. L. Swift, R. A. Siwek, and B. C. Wilson, "A control method for a nonlinear multivariable system: Application to interstitial laser hyperthermia," IEEE Trans. Biomed. Eng. 38, 891-898 (1992).

${ }^{10}$ H. Tanaka, K. Eno, H. Kato, and T. Ishida, "Possible application of noninvasive thermometry for hyperthermia using NMR," Nippon Acta Radiol. 41, 897-899 (1981).

${ }^{11}$ D. L. Parker, V. Smith, P. Sheldon, L. E. Crooks, and L. Fussel, "Temperature distribution measurements in two-dimensional NMR imaging," Med. Phys. 10, 321-325 (1983).

${ }^{12}$ R. J. Dickinson, A. S. Hall, A. J. Hind, and I. R. Young, "Measurements of changes in tissue temperature using MR imaging," J. Comput. Assist. Tomogr. 10, 468-472 (1988).

${ }^{13}$ D. J. Castro, R. E. Saxton, and L. J. Layfield, “Interstitial laser phototherapy assisted by magnetic resonance imaging: A new technique for monitoring laser-tissue interactions,' Laryngoscope 100, 541-547 (1990).

${ }^{14}$ A. S. Hall, M. V. Prior, J. W. Hand, I. R. Young, and R. J. Dickinson, "Observation by MR imaging of in vivo temperature changes induced by radio frequency hyperthermia," J. Comput. Assist. Tomogr. 3, 430-436 (1990).

${ }^{15}$ A. R. Bleier, F. A. Jolesz, M. S. Cohen, R. M. Weisskoff, J. J. Dalcanton, N. Higuchi, D. A. Feinberg, B. R. Rosen, and R. C. McKinstry, "Real time magnetic resonance imaging of laser-tissue interactions," Magn. Reson. Med. 21, 132-137 (1991).

${ }^{16} \mathrm{R}$. Matsumoto, K. Oshio, and F. A. Jolesz, “T1-weighted MR monitoring for interstitial laser- and freezing-induced ablation in the liver,' J. Magn. Reson. Imag. 2, 555-562 (1992).

${ }^{17}$ Y. Zhang, T. V. Samulski, W. T. Jonies, J. Matiello, R. L. Levin, and D. LeBihan, "On the accuracy of noninvasive thermometry using molecular diffusion magnetic resonance imaging," Int. J. Hyperthermia 8, 263-274 (1992).

${ }^{18}$ M. D. Matsumoto, R. V. Mulkern, S. G. Hushek, and F. A. Jolesz, “Tissue temperature monitoring for thermal interventional therapy: Comparison of T1-weighted MR sequences," J. Magn. Reson. Imag. 4, 65-70 (1994).

${ }^{19} \mathrm{~K}$. Kuroda et al., "Temperature mapping using water proton chemical shift obtained with 3D-MRSI: Feasibility in vivo," Magn. Reson. Med. 35, 35-40 (1996).

${ }^{20} \mathrm{Y}$. Ishihara et al. "A precise and fast temperature mapping using water proton chemical shift," Magn. Reson. Med. 34, 814-823 (1995).

${ }^{21}$ J. DePoorter, "Noninvasive MRI thermometry with the proton resonance frequency method: Study of susceptibility effects," Magn. Reson. Med. 34, 359-367 (1995).

${ }^{22}$ J. DePoorter, C. De Wagter, Y. De Deene, C. Thomsen, F. Ståhlberg, and E. Achten, "Noninvasive MRI thermometry with the proton resonance frequency (PRF) method: In vivo results in human muscle," Magn. Reson. Med. 33, 74-81 (1995).

${ }^{23}$ J. DePoorter, C. De Wagter, Y. De Deene, C. Thomsen, F. Ståhlberg, and E. Achten, "The proton-resonance-frequency-shift method compared with molecular diffusion for quantitative measurement of two- 
dimensional time-dependent temperature distribution in a phantom," J. Magn. Reson. B 103, 234-241 (1994).

${ }^{24}$ F. Franconi, F. Sequin, C. B. Sonier, A. Le Paper, and S. Akoka, "T1 mapping from spin echo and stimulated echoes," Med. Phys. 22, 17631769 (1995).

${ }^{25} \mathrm{~J}$. C. Hindman, "Proton resonance shift of water in gas and liquid states," J. Chem. Phys. 44, 4582-4592 (1966).

${ }^{26}$ W. G. Schneider, H. J. Bernstein, and J. A. Pople, "Proton magnetic resonance chemical shift of free (gaseous) and associated (liquid) hydride molecules," J. Chem. Phys. 28, 601-607 (1958).

${ }^{27}$ S. G. Bown, "Phototherapy of tumours," World J. Surg. 7, 700-709 (1983).

${ }^{28}$ R. A. Tracz, D. R. Wyman, P. B. Little, R. A. Towner, W. A. Stewart, S. W. Schatz, P. W. Pennock, and B. C. Wilson, "Magnetic resonance imaging of interstitial laser photocoagulation in brain," Lasers Surg. Med. 12, 165-173 (1992).

${ }^{29}$ C. T. Coughlin, E. B. Douple, J. W. Strohbehn, W. L. Eaton, B. S. Tremblay, and T. Z. Wong, "Interstitial hyperthermia in combination with brachytherapy,"' Radiology 148, 285-288 (1983).

${ }^{30}$ C. J. Diederich, G. Sherwin, C. Adams, and P. R. Stauffer, "Evaluation of a multielement microwave applicator for hyperthermia," Proceedings of the 9th International Conference on Radiation Research, Toronto, Canada, 1991 (unpublished), p. 194.

${ }^{31}$ J. Delannoy, D. LeBihan, D. I. Hoult, and R. L. Levin, "Hyperthermia system combined with a magnetic resonance imaging unit," Med. Phys. 17, 855-860 (1990).

${ }^{32} \mathrm{~J}$. W. Hunt, "Principles of ultrasound use for generating localized hyperthermia," in An Introduction to the Practical Aspects of Clinical Hyperthemia, edited by S. W. Field and J. W. Hand (Taylor and Francis, New York, 1990), pp. 371-422.

${ }^{33}$ K. Hynynen and K. L. Davis, "Small cylindrical ultrasound sources for induction of hyperthermia via body cavities or interstitial implants," Int. J. Hyperthermia 9, 263-274 (1993).
${ }^{34}$ C. J. Diederich, "Ultrasound applicators with integrated catheter-cooling for interstitial hyperthermia: Theory and preliminary measurements," Int. J. Hypertherm. 12, 279-297 (1996).

${ }^{35}$ I. A. Vitkin, J. A. Moriarty, R. S. Hinks, W. Kucharczyk, B. C. Wilson, and M. D. Sherar, "Magnetic resonance imaging of temperature changes during therapeutic interstitial microwave heating: a phantom study," COMP/CCPM Proceedings of 41st Annual General Meeting Montreal, Canada, 1995 (unpublished), pp. 51 and 52; see also I. A. Vitkin, J. A. Moriarty, R. S. Hinks, W. Kucharczyk, B. C. Wilson, and M. D. Sherar, "Magnetic resonance imaging of temperature changes during microwave heating: a phantom study," Proceedings of the Annual SMR Meeting, Nice, France, 1995 (unpublished), p. 500.

${ }^{36}$ H.-J. Schwarzmaier and T. Kahn, "Magnetic resonance imaging of microwave induced tissue heating," Magn. Reson. Med. 33, 729-731 (1995).

${ }^{37}$ A. Surowiec, P. N. Shrivastava, M. Astrahan, and Z. Petrovich, “Utilization of layered polyacrylamide phantom for evaluation of hyperthermia applicators,'” Int. J. Hyperthermia 8, 795-807 (1992).

${ }^{38}$ M. J. Bronskill and S. J. Graham, "NMR characteristics of tissue," in The Physics of MRI: 1992 AAPM Summer School Proceedings, edited by P. Sprawls and M. J. Bronskill, Med. Phys. Monograph No. 21 (American Institute of Physics, New York, 1993), pp. 32-55.

${ }^{39}$ A. S. Gladman, T. Easty, M. Joy, and M. D. Sherar, "Effect of thermal conduction on the accuracy of infrared-based SAR measurement for the evaluation of interstitial microwave hyperthermia applicators," in Canadian Medical and Biological Engineering Society Conference Proceedings: 1996 (CMBES, Gloucester, Canada, 1996), pp. 64 and 65.

${ }^{40}$ J. Thibault, "Comparison of nine three dimensional numerical methods for the solution of the heat diffusion equation," Num. Heat Trans. 8, 281-298 (1985).

${ }^{41}$ T. P. Ryan, "Comparison of six microwave antennas for hyperthermia treatment of cancer: SAR results for single antennas and arrays," Int. J. Radiat. Oncol. Biol. Phys. 21, 403-413 (1991). 